\title{
FDA Critical Path Initiatives: Opportunities for Generic Drug Development
}

\author{
Robert A. Lionberger ${ }^{1,2}$
}

Received 15 August 2007; accepted 17 January 2008; published online 20 February 2008

\begin{abstract}
FDA's critical path initiative documents have focused on the challenges involved in the development of new drugs. Some of the focus areas identified apply equally to the production of generic drugs. However, there are scientific challenges unique to the development of generic drugs as well. In May 2007, FDA released a document "Critical Path Opportunities for Generic Drugs" that identified some of the specific challenges in the development of generic drugs. The key steps in generic product development are usually characterization of the reference product, design of a pharmaceutically equivalent and bioequivalent product, design of a consistent manufacturing process and conduct of the pivotal bioequivalence study. There are several areas of opportunity where scientific progress could accelerate the development and approval of generic products and expand the range of products for which generic versions are available, while maintaining high standards for quality, safety, and efficacy. These areas include the use of quality by design to develop bioequivalent products, more efficient bioequivalence methods for systemically acting drugs (expansion of BCS waivers, highly variable drugs), and development of new bioequivalence methods for locally acting drugs.
\end{abstract}

KEYWORDS: bioequivalence; critical path initiative; generic drugs.

\section{INTRODUCTION}

In 2004, FDA introduced the Critical Path Initiative (1) that focused attention on the challenges involved in the development of new drugs. After two years, a follow up set of specific opportunities was created based on response to the original initiative $(2,3)$. While some areas apply equally to the production of generic drugs, there are scientific challenges unique to the development of generic drugs. In May 2007, FDA released a document "Critical Path Opportunities for Generic Drugs" that identified some of the specific challenges in the development of generic drugs (4). The goal of this document was to bring these challenges to the attention of interested parties and identify opportunities for collaborative solutions.

The need to address generic drug development was motivated by the large and growing use of generic drugs. Generic drugs represented 66.4 percent of the total prescription doses sold in 2004, compared with 61 percent in 2001 (5). Efforts to encourage efficient pharmaceutical manufacturing processes and quality by design will only have maximum impact when they are applied to generic drugs. While the focus of the critical path initiative for generic drugs is on generic drug development for the US, the scientific challenges

Opinions expressed in this manuscript are those of the author and do not necessarily reflect the views or policies of the FDA.

\footnotetext{
${ }^{1}$ Office of Generic Drugs, Food and Drug Administration, 7519 Standish Place, Rockville, Maryland 20850, USA.

${ }^{2}$ To whom correspondence should be addressed. (e-mail: robert. lionberger@fda.hhs.gov)
}

and their resolutions may have impact on ensuring generic product quality in other markets.

The impact of generic competition is felt immediately upon the approval of the first generic products. The Office of Generic Drugs (OGD) website contains an analysis of IMS sales data (wholesale prices) for single-ingredient brand name and generic drug products sold in the U.S. from 1999 through 2004 (6). This analysis indicates that the appearance of a second generic manufacturer reduces the average generic price to nearly half the brand name price. As additional generic manufacturers market the product, the prices continue to fall. For products that attract a large number of generic manufacturers, the average generic price falls to $20 \%$ of the branded price.

These results show the value to the consumer of having multiple generic drug products competing in the market. However, there are still products and product categories for which there is limited or no generic competition, even though all patent and exclusivity protection for the reference product has expired. In order to understand this we need to take a more detailed look at the generic drug development process and identify the scientific reasons for the lack of competition. This investigation lead to the critical path opportunities identified in the May 2007 FDA report.

\section{GENERIC DRUG DEVELOPMENT PROCESS}

The steps involved in the development of a potential generic product can be described in Fig. 1: 


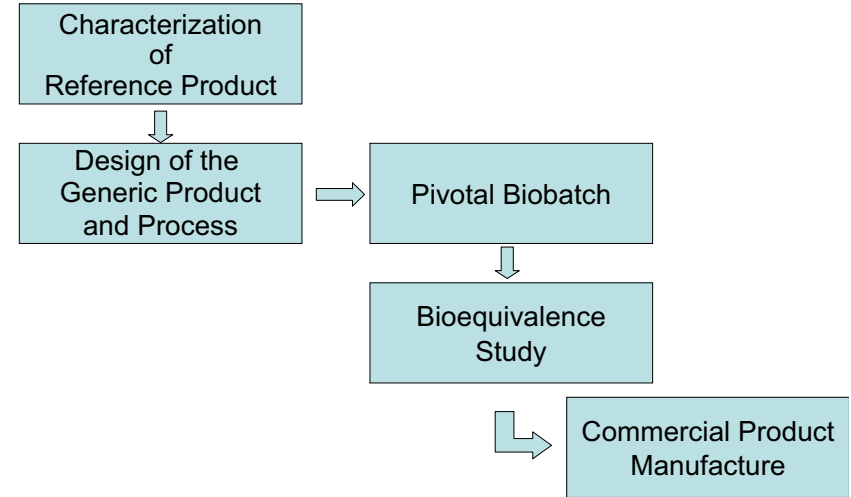

Fig. 1. Development of a generic drug product

Unlike the case for new drugs, the risk of total failure is low because the safety and efficacy of the active ingredient have already been established, but cost efficiency is more important because of much lower profit margins and competition with other generic manufacturers. Time efficiency is important to both generic and new drug manufacturers with neither wanting unanswered scientific questions to slow the progress of products through the development process. Another aspect to efficient drug development is to use scientific understanding to limit unnecessary human testing of drugs. Bottlenecks to the efficient development (in the senses of resources, time, and appropriate use of human subjects) of a generic products can occur at every stage in the development process.

In the characterization stage, some generic products are challenges to develop because of the complexity of the reference product. Complex reference products may include drug substances with many potentially active molecules. Molecular diversity can arise in products with a natural source origin or even in synthetic molecules that are polydisperse mixtures. Other challenging reference products include products with complex supra-molecular structures such as iron complexes and products containing liposomes. The use of nanotechnology in drug delivery will lead to even more complex pharmaceutical structures to characterize. To make a copy of these products, an ANDA sponsor may need better characterization than the originator.

In the formulation development stage, generic formulations may be developed that do not pass the bioequivalence study or meet other requirements and formulation development must be repeated. These failures can sometimes be linked to the inability of dissolution or lack of an IVIVC to evaluate proposed formulations and processes during development. For new or complex dosage forms the formulation and product development stage can fail because of an inability to identify the critical quality attributes of new or complex dosage forms. Inhalers are complex combination of device and formulation and many are breath actuated and thus must perform equivalently to the reference product over a range of flow rates. Transdermal products must be bioequivalent to the RLD in terms of the rate and extent of drug delivery, but must also be equivalent in terms of adhesion, irritation and sensitization to the RLD when applied to the skin. Concern about the interaction of modified release formulations with alcohol (7), has introduced a new potentially critical quality attribute for some drug products.
The type of bioequivalence study required can determine whether the development process is economically and scientifically feasible. Expensive, extensive or unpredictable BE tests can limit the development of a generic product. A highly variable drug may require a large number of subjects using the usual FDA recommended study. However, the biggest limitation to generic competition is when FDA recommends a clinical endpoint bioequivalence study. Many generic companies do not develop products that need clinical endpoint bioequivalence studies because of the relative cost, time and risk of failure involved. Clinical endpoint studies can add up to $\$ 2-6$ million to the cost ${ }^{1}$. Thus, when expensive and risky (to the sponsor) clinical endpoint trials are needed to establish bioequivalence, the number of generic competitors is reduced significantly.

Finally, a generic drug that demonstrates bioequivalence to the reference product can be delayed in reaching the market because of the final scale-up step in the generic drug development process. Problems on scale up include wasted commercial batches, failure to meet specifications, and process variability. These problems may require the sponsor to reformulate product or revise the process.

\section{CRITICAL PATH OPPORTUNITIES}

Based on this analysis of generic drug development, there are several areas of opportunity where scientific progress could accelerate the development and approval of generic products and expand the range of products for which generic versions are available, while maintaining high standards for quality, safety, and efficacy. FDA presented these opportunity areas in its May 2007 document (4).

- Improve the science underlying quality by design for the development and manufacture of generic drug products.

- Improve the efficiency of current methods for assessment of bioequivalence of systemically acting drugs including products that use complex and novel drug delivery technologies.

- Develop methods for the assessment of bioequivalence of locally acting drugs such as topical and inhalation products.

- Develop methods for characterizing complex drug substances and products.

Figure 2 indicates how these opportunity areas link into the generic drug development process. Progress in these areas will accelerate approval of generic drug products. More importantly, it will expand the range of products for which generic versions are available, while maintaining high standards for quality, safety, and efficacy. Methods for equivalence based on sound science build the confidence of health care providers, patients, and the public that generic products are equivalent to innovator products.

\footnotetext{
${ }^{1}$ We use an estimate of $\$ 10,000$ per subject as an estimate based on costs of phase III trials reported in the literature $[8,9,10]$. We assume a study size of 200-600 subjects.
} 


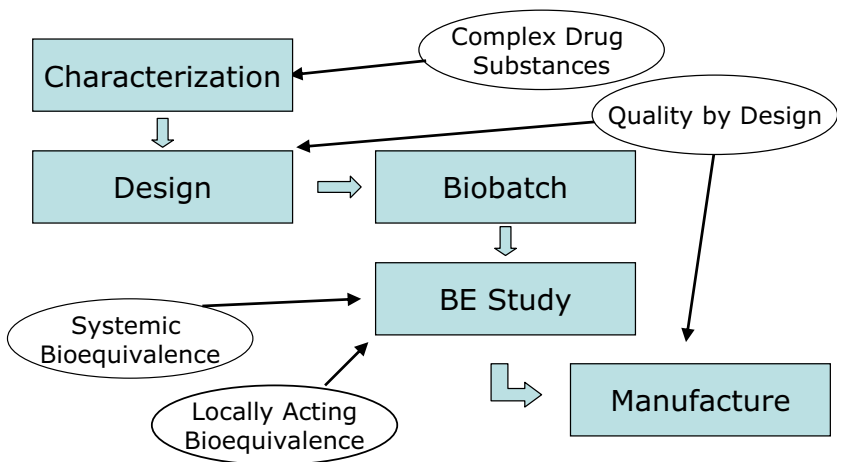

Fig. 2. Critical Path areas linked to development of generic drug products

\section{BIOEQUIVALENCE ON THE CRITICAL PATH}

In this paper we focus on the critical path opportunities that are connected to the evaluation of bioequivalence.

- Quality by design tools to aid in the design and manufacture of bioequivalent products

- Bioequivalence of systemic drugs

- Bioequivalence of locally acting drugs

\section{Quality by Design for Bioequivalence}

Quality by design (QbD) is often discussed in the context of CMC submission or manufacturing process understanding. Especially for generic drugs, which have very specific performance goals (bioequivalent to the RLD); QbD tools to aid in the design and manufacture of bioequivalent products are desirable.

QbD identifies characteristics that are critical to quality from a safety and efficacy perspective, translates them into the attributes that the drug product should possess, and establishes how the critical process parameters can be varied to consistently produce a drug product with the desired attributes. This is achieved by establishing a relationship between formulation and manufacturing process variables (including drug substance and excipient attributes and process parameters) and product quality, and identifying the sources of product variability.

To use QbD to develop a product that is bioequivalent to a reference product, a generic sponsor must have an understanding of what attributes of the formulation and manufacturing process have the potential to change the bioavailability of a particular active ingredient.

Current formulation development strategies for generic drugs are mainly empirical. Within a QbD process, modeling and simulation using improved absorption models could help a generic sponsor identify the formulation and manufacturing variables to be adjusted to make the product bioequivalent. Based on the properties of the active ingredient (solubility, permeability, interactions with metabolic enzymes and transporter proteins), integrated absorption models could be used to estimate (or in some cases predict) the relation between an in vivo dissolution or release rate and the pharmacokinetics parameters that are used to evaluate bioequivalence. The current state of modeling and simulation tools does not allow this type of formulation development from a mechanistic understanding. However, progress could be made through looking at data from a wide variety of drugs/formulations.

Modeling and simulation can aid generic developers in determining what drug release profile is needed to provide bioequivalence to the reference product. Then the challenge to the generic developer becomes the selection of excipients and design of a formulation and release mechanism that will provide the intended in vivo release profile. Mechanistic understanding of how physical properties of drug substance and excipients affect drug product performance can enable rational choices of excipients and reduce the number of experimental formulations that need to be produced.

Because it is usually not feasible to test every trial formulation in an in vivo study, better in vitro/in vivo correlations (IVIVC) will give better feedback within an iterative design process. When an in vitro dissolution test can be related to the in vivo dissolution of a product, a generic sponsor would have an efficient tool to evaluate different formulations and select the optimal formulation for use in the pivotal bioequivalence study. However, the correlation of dissolution testing with in vivo performance varies from product to product. FDA's data from dissolution tests and pharmacokinetic studies could help external collaborators develop and test models capable of predicting the connection between dissolution and bioavailability/bioequivalence.

FDA's guidance on the establishment of IVIVCs (11) has not been used extensively by ANDA sponsors because it requires the performance of multiple in vivo pharmacokinetic studies with different formulations to establish an IVIVC. This guidance then allows the IVIVC to be used for regulatory purposes in place of in vivo pharmacokinetic studies. This process has not been of value to ANDA sponsors. However, an understanding (even a theoretical one) of the relation between in vitro tests and in vivo performance is very valuable to ANDA sponsors during the product development process. It is part of the critical path opportunity to identify and resolve the scientific issues that limit the use of IVIVCs by ANDA sponsors.

\section{Bioequivalence for Systemically Acting Drugs}

For systemically acting drugs, the critical path goal is to increase the efficiency of a process that already is providing safe and effective generic drugs to the public. The use of the Biopharmaceutics Classification System (BCS) $(12,13)$ in the development of both new and generic drug products is an example of how critical path approaches can improve the efficiency of pharmaceutical development and improve product quality. Expanding the use of biowaivers in appropriate cases and improving dissolution methods are efficient ways to accelerate the approval and development of high quality generic products. FDA has also identified other issues for which resolution could lead to more efficient bioequivalence testing. Many of these areas were discussed in scientific detail at the May 2007 AAPS BE, BCS and Beyond workshop and examples included

- Expansion of BCS biowaivers to some class II and class III drugs (14)

- Development of biorelevant dissolution $(15,16,17)$

- Mechanistic understanding of food effects

- Bioequivalence methods for highly variable drugs (18) 


\section{Bioequivalence Methods for Locally Acting Drugs}

Bioequivalence of locally acting drugs is a long standing challenge to the generics program, has the biggest impact on generic competition, and raises the most challenging scientific issues. Locally acting drugs are primarily found in the following categories: topical dermatological products, orally inhaled and nasal drug products, and GI acting drug products. They often require exploration of alternative bioequivalence methods because plasma concentration profiles of these products are not always appropriate surrogates of pharmacological activity. There was little discussion of these issues at the May 2007 AAPS BE, BCS and Beyond workshop. It is the intention of the "Critical Path Opportunities for Generic Drugs" report to attract the attention of leading scientists to address these complex challenges.

Difficulty in demonstrating bioequivalence of local acting drugs also has consequences to NDA sponsors across a product's life cycle. In initial development, process scale-up and optimization become difficult if there is not a method to compare pre and post change products. After approval, manufactures are reluctant to make major manufacturing improvements if a clinical study is required to validate the changes.

The selection of the bioequivalence method for a locally drug is based on product specific factors and a scientific understanding of the products' mechanism of action. FDA's regulation 21 CFR 320.24 lists approaches that are acceptable for determining the bioavailability or bioequivalence of a drug product. All of the approaches listed have been used for bioequivalence of locally acting drugs. A 2003 addition to the Federal Food Drug and Cosmetic Act at Section 505(j)(8)(A) (ii)indicates that "For a drug that is not intended to be absorbed into the bloodstream, the Secretary may assess bioavailability by scientifically valid measurements intended to reflect the rate and extent to which the active ingredient or therapeutic ingredient becomes available at the site of drug action".

\section{Clinical Endpoint Bioequivalence Study}

Even though 21 CFR 320.24 indicates that "This approach is the least accurate, sensitive, and reproducible of the general approaches for measuring bioavailability or demonstrating bioequivalence." for many locally acting drugs, FDA recommends a bioequivalence study with clinical endpoints. It has become the fall-back method because selection of one of the other approaches depends on scientific understanding for a particular drug product, but all drugs have a clinical endpoint used to support their initial approval.

A bioequivalence study with clinical endpoints will use a product-specific clinical indication recommended by FDA. Patients in the study would be given the test product, the reference product, and/or a placebo. The placebo arm ensures that the study and its conduct are sufficiently sensitive to differences between treatments. If the reference product is labeled for multiple indications, then the indication that is most sensitive to difference in local delivery of drug is usually preferred. The ability of clinical endpoint bioequivalence studies to detect differences in formulation performance between products depends on the exposure-response relation for the particular drug and indication.
Most clinical endpoint bioequivalence studies have a dichotomous endpoint; the treatment either succeeds or fails. To decide if the test product is bioequivalent to the reference, the success proportion for each treatment is calculated, and if the $90 \%$ confidence interval for the difference in success is within $-20 \%$ to $+20 \%$, then the test product passes. For dichotomous endpoints, there is no meaning to betweensubject variability and generally studies must enroll approximately 200 to 600 subjects to ensure sufficient power.

Table I provides some example results of clinical studies used in support of ANDAs. All of these studies had three arms and used the difference in cure rate as the endpoint with an acceptable $90 \%$ confidence interval of -20 to +20 percentage points. The results show that even with relatively large numbers of patients in each study, the confidence intervals were close to the limits defined by FDA. This suggests that these studies were at a high risk of failure to demonstrate bioequivalence.

Because clinical endpoint bioequivalence studies can be larger than studies conducted in support of the initial $\mathrm{NDA}^{2}$ and the insensitivity of some clinical endpoints to formulation differences, there is much interest in alternate bioequivalence methods that are more efficient and more sensitive at detecting product differences.

\section{In Vitro Study}

In vitro bioequivalence studies have been used for some locally acting products. Examples include an in vitro binding assay for cholestyramine (20) and dissolution studies for some immediate release GI acting products that are high solubility. Open scientific questions remain for immediate release GI acting products that are low solubility and modified release GI acting products.

For nasal and inhalation products, in vitro equivalence tests are a critical aspect of evaluate the performance of the device. For solution products, in vitro tests alone demonstrate bioequivalence, while for suspension products the in vitro tests are combined with PK, PD, or clinical endpoint studies as needed to ensure equivalence local delivery (21).

The SUPAC-SS (22) guidance discusses the role of in vitro release testing for semi-solid dosage forms. The guidance states that "An in vitro release rate can reflect the combined effect of several physical and chemical parameters, including solubility and particle size of the active ingredient and rheological properties of the dosage form. In most cases, in vitro release rate is a useful test to assess product sameness between prechange and post-change products." The use of in vitro release tests is currently limited to evaluating changes in manufacturing process or scale-up by the same manufacturer and is not used for bioequivalence. However, when the test and the

\footnotetext{
${ }^{2}$ Approval of a new drug product requires that "adequate and wellcontrolled studies" demonstrate that the product is safe and effective. These clinical studies may either demonstrate superiority over placebo or non-inferiority over an active control that is approved for the same indication that is being sought for the new product. Demonstrating equivalence requires both that the study be sensitive enough to detect differences between products and that it show equivalent clinical efficacy between the products and thus can require a larger study.
} 
Table I. Example Clinical Endpoint Study Sizes for a Topical Antifungal Drug (19)

\begin{tabular}{llll}
\hline$N$ & \%Cure Test & \%Cure Ref & 90\%CI \\
\hline 728 & 50 & 48 & {$[-12,+16]$} \\
453 & 46 & 40 & {$[-8,+20]$} \\
447 & 29 & 27 & {$[-9,+13]$} \\
\hline
\end{tabular}

reference product have identical compositions, the only differences are in manufacturing and process scale.

\section{Pharmacokinetic Study}

For locally acting products plasma concentrations may be appropriate for use in a bioequivalence study when the plasma concentration reflects the delivery to the site of action. In the GI-acting category, pharmacokinetic bioequivalence studies have been used for mesalamine pro-drugs (23). For topical drugs applied to the skin the amount of drug that reached the systemic circulation from a topical dermatological products was typically not detectable. With advances in assays, many drugs can be detected and compared. For some topical products, such as lidocaine/prilocaine cream (24), pharmacokinetic studies have been accepted by FDA as evidence for bioequivalence. However, using a pharmacokinetic study requires that the area of drug application be similar between test and reference products.

\section{Pharmacodynamic Study}

Another approach to bioequivalence of locally acting drugs is to measure a pharmacodynamic effect of the drug. The pharmacodynamic endpoint can differ from the clinical effect of the product. It is generally needed to demonstrate the dose-response of the pharmacodynamic endpoint.

For topical corticosteroids, there is a 1995 FDA guidance (25) that describes a pharmacodynamic bioequivalence study $(26,27,28,29)$. As in all pharmacodynamic bioequivalence studies, it is necessary to establish sufficient sensitivity in the dose-response curve to detect differences between products. For the skin blanching study, the dose is varied by changing the amount of time the topical product is applied to the skin. A pilot study using a range of application times of the reference product is used to identify patients in whom skin blanching is sensitive to differences in application time. Then, these patients are used to compare the test and reference products.

For inhalation products, pharmacodynamic measures of lung function have been used in the approval of ANDAs for albuterol MDIs (30). These applications used histamine PC20 or FEV1 as the endpoints. Equivalence of local delivery using these endpoints was evaluated using a "dose-scale" method, in which the relative bioavailability is determined in terms of dose of the test formulation required to produce an equivalent PD response to the reference product (31).

\section{New Methods for Locally Acting Drugs}

In general, the alternatives to clinical endpoint studies are preferable because of sensitivity and efficiency, but the alternatives need to be scientifically justified. The 2007 critical path report (4) identifies possible research directions to address these problems that include:

- Molecular Level Imaging: Imaging techniques that can quantify the amount of drug at the site of action can be used to validate new in vitro tests or new biomarkers. In the past OGD, has rejected for direct use in bioequivalence testing any imaging technique that would require modification or labeling of the reference product. However, new methods that do not require product modification could be used directly as a bioequivalence method.

- In Vivo Sampling Methods: Direct sampling of target tissue (for example by microdialysis $(32,33,34,35)$ or tape stripping $(36,37,38,39,40,41,42,43,44,45)$ for dermatological products) could be used as a bioequivalence method when blood concentrations are not appropriate.

- Pharmacodynamic Measures: New pharmacodynamic measures (biomarkers) that are sensitive to local drug concentrations can be used for bioequivalence testing. When we discuss biomarkers for bioequivalence our goal is to identify biomarkers of local delivery. Other studies on the reference product have already established safety and efficacy. An example that is under investigation by FDA is exhaled $\mathrm{NO}$ as a marker of local delivery of an inhaled corticosteroid $(46,47,48,49)$. For use in a bioequivalence study a biomarker must have an exposure-response relation that allows sensitivity.

- Novel Pharmacodynamic Study Designs: Many asthma drugs have a very shallow dose-response curve and exhibit high within- and between-subject variability, requiring the use of a very large number of subjects in a pharmacodynamic equivalence test. Novel pharmacodynamic study designs that enable using a forced expiratory volume in 1 second (FEV1) endpoint in a crossover study would allow bioequivalence studies to be conducted using a much smaller number of subjects (50).

- Design of Bioequivalence Trials with Clinical Endpoints: New approaches to design clinical trials with the goal of more efficiently demonstrating equivalence could be investigated. Sometimes, pharmacokinetic methods cannot be used to assess generics for approval, and we have to use trials with clinical endpoints or pharmacodynamic measures. In such cases, there is interest in better understanding when noninferiority trial designs can be used $(51,52,53)$.

- Particle Size Measurement for Suspension Bioequivalence: For many locally acting products that contain suspended drug, in vivo bioequivalence studies are requested because the particle size distribution in the suspensions cannot be directly compared (21). Some of the challenges in developing these methods are to distinguish drug particles from other particles in the product and to ensure that the measurement process does not itself affect the particle size. For nonspherical particles there are other challenges (54). Once this measurement is possible with sufficient accuracy and precision, a demonstration of equivalent particle size in combination with other relevant characterizations of particle properties could be used 
to support a waiver of in vivo bioequivalence studies as is done for solution products.

\section{CONCLUSIONS}

The FDA role in the critical path initiative is to identify the challenges as it did in 2004 for new drugs and in 2007 for generic drugs. This initial report on critical path opportunities for generic drugs is intended to stimulate discussion and comments. In response to this input from interested parties including industry, academia, other government agencies (NIH, NSF, NIST), and the public, FDA intends to participate in collaborative solutions to these critical path challenges.

A challenge in bringing together consortia that involve industrial sponsors is that the generic sponsors are in direct competition with each other for the same product (this differs from new drugs where companies may compete with different products for the same clinical indication or therapeutic area). Thus an ANDA sponsor that invests efforts to address one of these issues will be aiding their competitors who may gain the advantage of scientific advances without any investment. This may require the design of new types of consortia with tangible benefits for participation.

A potential solution is for consortia to establish domains of "pre-competitive knowledge" that can be shared freely and openly (55). For example, a consortium may be assembled to develop and present evidence that convinces FDA to establish a new bioequivalence method for a particular class of products. Each ANDA sponsor would then develop their own product and use this method to establish bioequivalence to the reference product.

Solutions to many of these critical path challenges could result in large savings to the public. For example, for a drug with $\$ 500$ million dollars/year in sales that has no generic competition due to scientific challenges, development of a new bioequivalence method could save the public hundreds of million dollars per year. Even an investment as large as $\$ 10$ million to establish a new bioequivalence method for this product category, would give a large return on investment to the public. However, any individual generic manufacturer that invested $\$ 10$ million in a bioequivalence method for a product might never be able to recover that investment because of competition with other generic manufactures. Thus, identifying the source for resources to apply to these critical path opportunities is a challenge.

\section{REFERENCES}

1. FDA, Challenge and Opportunity on the Critical Path to New Medicinal Products March 2004, Available at: http://www.fda.gov/ oc/initiatives/criticalpath/whitepaper.pdf. Accessed 1/15/2008.

2. FDA, Critical Path Opportunities Report March 2006, Available at: http://www.fda.gov/oc/initiatives/criticalpath/reports/opp_report. pdf. Accessed 1/15/2008.

3. FDA, Critical Path Opportunities List March 2006, Available at: http://www.fda.gov/oc/initiatives/criticalpath/reports/opp_list.pdf. Accessed 1/15/2008.

4. FDA, Critical Path Opportunities for Generic Drugs May 2007 , Available at: http://www.fda.gov/oc/initiatives/criticalpath/ reports/generic.html. Accessed 1/15/2008.
5. Gottlieb S. Speech before annual generic drug forum. FDA 2006, Available at: http://www.fda.gov/oc/speeches/2006/generic drug0407.html. Accessed 1/15/2008.

6. OGD. Generic competition and drug prices. FDA 2005, Available at: http://www.fda.gov/cder/ogd/generic_competition.htm. Accessed 1/15/2008.

7. Meyer R. Clinical relevance of alcohol-induced dose dumping. at: Advisory Committee For Pharmaceutical Science October 2005 Available at: http://www.fda.gov/ohrms/dockets/ac/05/slides/ 2005-4187S2_01_Meyer.ppt. Accessed 1/15/2008.

8. S. Ustunel. How to put an accurate value on biotech firms. Prof. Invest. 2005:19-25.

9. A Guidance Document for Implementing Effective Cancer Clinical Trials Executive Summary Version 1.2 June 2005, Available at: http://www.cancertrialshelp.org/pdf/cchange.pdf. Accessed 1/15/2008.

10. E. Miller. Clinical investigators the endangered species revisited. J. Am. Med. Assoc. 286:845-846 (2001).

11. Center for Drug Evaluation and Research. Extended release oral dosage forms: Development, evaluation, and application of in vitro/in vivo correlations. FDA 1997, Available at: http://www. fda.gov/cder/guidance/1306fnl.pdf. Accessed 1/15/2008.

12. G. L. Amidon, H. Lennernas, V. P. Shah, and J. R. Crison. A theoretical basis for a biopharmaceutic drug classification: the correlation of in vitro drug product dissolution and in vivo bioavailability. Pharm. Res. 12:413-420 (1995).

13. Guidance for Industry: Waiver of In-vivo Bioavailability and Bioequivalence Studies for Immediate Release Solid Oral Dosage Forms Based on a Biopharmaceutics Classification System 2001, Available at: http://www.fda.gov/cder/guidance/3618fnl.pdf. Accessed 1/15/2008.

14. J. E. Polli, L. X. Yu J. A. Cook, et al. Summary workshop report: Biopharmaceutics classification system-implementation challenges and extension opportunities. J. Pharm. Sci. 93:1374-1381 (2005).

15. E. Nicolaides, E. Galia, C. Efthymiopoulos, J. B. Dressman, and C. Reppas. Forecasting the in vivo performance of four low solubility drugs from their in vitro dissolution data. Pharm. Res. 16:1876-1882 (1999).

16. M. Vertzoni, J. Dressman, J. Butler, J. Hempenstall, and C. Reppas. Simulation of fasting gastric conditions and its importance for the in vivo dissolution of lipophilic compounds. Eur. J. Pharm. Biopharm. 60:413-417 (2005).

17. L. Kalantzi, K. Goumas, V. Kalioras, B. Abrahamsson, J. B. Dressman, and C. Reppas. Characterization of the human upper gastrointestinal contents under conditions simulating bioavailability/bioequivalence studiesPharm. Res:.:23:165-176 (2006).

18. S. H. Haidar, B. Davit, M. L. Chen, et al. Bioequivalence approaches for highly variable drugs and drug products. Pharm. Res. 2007. Epub September 22007.

19. R. Lionberger. Topical bioequivalence update. at: Advisory Committee for Pharmaceutical Science April 2004 Available at: http://www.fda.gov/ohrms/dockets/AC/04/slides/4034S2_12_ Lionberger.ppt. Accessed 1/15/2008.

20. Center for Drug Evaluation and Research. Interim guidance: cholestyramine powder in vitro bioequivalence 1993, Available at: http://www.fda.gov/CDER/GUIDANCE/cholesty.pdf. Accessed $1 / 15 / 2008$

21. Center for Drug Evaluation and Research. Draft guidance for industry: Bioavailability and bioequivalence studies for nasal aerosols and nasal sprays for local action. FDA Apr 2003, Available at: http://www.fda.gov/CDER/GUIDANCE/5383DFT. pdf. Accessed 1/15/2008.

22. Center for Drug Evaluation and Research. Guidance for industry: Nonsterile semisolid dosage forms scale-up and postapproval changes: Chemistry, manufacturing, and controls; in vitro release testing and in vivo bioequivalence documentation. FDA May 1997, Available at: www.fda.gov/cder/guidance/ 1447fnl.pdf. Accessed 1/15/2008.

23. OGD. Approval package for ANDA 040349. FDA 2002, Available at: http://www.fda.gov/cder/foi/nda/2002/040349.pdf. Accessed 1/15/2008.

24. OGD. Approval package for ANDA 076453. FDA 2003, Available at: http://www.fda.gov/cder/foi/nda/2003/076453.pdf. Accessed 1/15/2008. 
25. Center for Drug Evaluation and Research. Topical dermatological corticosteriods: in vivo bioequivalence. FDA 1995, Available at: http://www.fda.gov/cder/guidance/old098fn.pdf. Accessed 1/ $15 / 2008$

26. A. W. McKenzie, and R. B. Stoughton. Method for comparing percutaneous absorption of steroids. Arch. Dermatol. 86:608-610 (1962).

27. E. W. Smith, J. M. Haigh, and R. B. Walker. Analysis of chromameter results obtained from corticosteroid-induced skin blanching. I: Manipulation of data. Pharm. Res. 15:280-285 (1998).

28. L. K. Pershing, L. Lambert, E. D. Wright, V. P. Shah, and R. L. Williams. Topical $0.050 \%$ betamethasone dipropionate. Pharmacokinetic and pharmacodynamic dose-response studies in humans. Arch. Dermatol. 130:740-747 (1994).

29. G. J. Singh, W. P. Adams L. J. Lesko, et al. Development of in vivo bioequivalence methodology for dermatologic corticosteroids based on pharmacodynamic modeling. Clin. Pharmacol. Ther. 66:346-357 (1999).

30. OGD. Approval package for ANDA 073045. FDA 1997, Available at: http://www.fda.gov/cder/foi/anda/97/073045_albuterol_ toc.htm. Accessed 1/15/2008.

31. M. L. Chen, V. Shah R. Patnaik, et al. Bioavailability and bioequivalence: an FDA regulatory overview. Pharm. Res. 18:1645-1650 (2001).

32. E. Schnetz, and M. Fartasch. Microdialysis for the evaluation of penetration through the human skin barrier-a promising tool for future research. Eur. J. Pharm. Sci. 12:165-174 (2001).

33. M. Kreilgaard. Assessment of cutaneous drug delivery using microdialysis. Adv. Drug Deliv. Rev. 54(Suppl 1):S99-121 (2002).

34. M. Kreilgaard, M. J. Kemme, J. Burggraaf, R. C. Schoemaker, and A. F. Cohen. Influence of a microemulsion vehicle on cutaneous bioequivalence of a lipophilic model drug assessed by microdialysis and pharmacodynamics. Pharm. Res. 18:593-599 (2001).

35. E. Benfeldt, S. H. Hansen, A. Volund, T. Menne, and V. P. Shah. Bioequivalence of topical formulations in humans: evaluation by dermal microdialysis sampling and the dermatopharmacokinetic method. J. Invest. Dermatol. 127:170-178 (2007).

36. A. Bunge. Dermatopharmacokinetics: Improvement of methodology for assessing bioequivalence of topical products: presentation to FDA advisory committee for pharmaceutical science, October 2003. FDA 2003, Available at: http://www.fda.gov/ohrms/ dockets/ac/03/slides/3996S2_07_Bunge.pdf. Accessed 1/15/2008.

37. L. K. Pershing, S. Bakhtian, C. E. Poncelet, J. L. Corlett, and V. P. Shah. Comparison of skin stripping, in vitro release, and skin blanching response methods to measure dose response and similarity of triamcinolone acetonide cream strengths from two manufactured sources. J. Pharm. Sci. 91:1312-1323 (2002).

38. V. P. Shah. Progress in methodologies for evaluating bioequivalence of topical formulations. Am. J. Clin. Dermatol. 2:275-280 (2001).

39. L. K. Pershing. New approaches to assess topical corticosteroid bioequivalence: pharmacokinetic evaluation. Int. J. Dermatol. 31 (Suppl 1):14-20 (1992).
40. L. K. Pershing, J. L. Nelson, J. L. Corlett, S. P. Shrivastava, D. B. Hare, and V. P. Shah. Assessment of dermatopharmacokinetic approach in the bioequivalence determination of topical tretinoin gel products. J. Am. Acad. Dermatol. 48:740-751 (2003).

41. K. Tojo, and A. C. Lee. A method for predicting steady-state rate of skin penetration in vivo. J. Invest. Dermatol. 92:105-108 (1989).

42. K. Moser, K. Kriwet, C. Froehlich, Y. N. Kalia, and R. H. Guy. Supersaturation: enhancement of skin penetration and permeation of a lipophilic drug. Pharm. Res. 18:1006-1011 (2001).

43. F. Pirot, Y. N. Kalia, A. L. Stinchcomb, G. Keating, A. Bunge, and R. H. Guy. Characterization of the permeability barrier of human skin in vivo. Proc. Natl. Acad. Sci. U. S. A. 94:1562-1567 (1997).

44. Y. N. Kalia, I. Alberti, A. Naik, and R. H. Guy. Assessment of topical bioavailability in vivo: the importance of stratum corneum thickness. Skin Pharmacol. Appl. Skin. Physiol. 14 (Suppl 1):82-86 (2001).

45. S. J. Bashir, A. L. Chew, A. Anigbogu, F. Dreher, and H. I. Maibach. Physical and physiological effects of stratum corneum tape stripping. Skin Res. Technol. 7:40-48 (2001). Clinical Trial.

46. A. M. Wilson, and B. J. Lipworth. Dose-response evaluation of the therapeutic index for inhaled budesonide in patients with mild-to-moderate asthma. Am. J. Med. 108:269-75 (2000).

47. P. E. Silkoff, P. McClean, M. Spino, L. Erlich, A. S. Slutsky, and N. Zamel. Dose-response relationship and reproducibility of the fall in exhaled nitric oxide after inhaled beclomethasone dipropionate therapy in asthma patients. Chest. 119:1322-8 (2001).

48. S. A. Kharitonov, and P. J. Barnes. Effects of corticosteroids on noninvasive biomarkers of inflammation in asthma and chronic obstructive pulmonary disease. Proc. Am. Thorac. Soc. 1:191-9 (2004).

49. Z. Zietkowski, I. Kucharewicz, and A. Bodzenta-Lukaszyk. The influence of inhaled corticosteroids on exhaled nitric oxide in stable chronic obstructive pulmonary disease. Respir. Med. 99:816-24 (2005).

50. R. C. Ahrens, M. E. Teresi, S. H. Han, D. Donnell, J. A. V. Burgt, and C. R. Lux. Asthma stability after oral prednisone: a clinical model for comparing inhaled steroid potency. Am. J. Resp. Crit. Care Med. 164:1138-45 (2001).

51. V. W. Steinijans, M. Neuhäuser, and F. Bretz. Equivalence concepts in clinical trials. Eur. J. Drug Metab. Pharmacokinet. 25:38-40 (2000).

52. L. L. Laster, and M. F. Johnson. Non-inferiority trials: the 'at least as good as' criterion. Stat. Med. 22:187-200 (2003).

53. Z. Zhang. Non-inferiority testing with a variable margin. Biom. J. 48:948-65 (2006).

54. B. Y. Shekunov, P. Chattopadhyay, H. H. Y. Tong, and A. H. L. Chow. Particle size analysis in pharmaceutics: principles, methods and applications. Pharm. Res. 24:203-227 (2007).

55. D. Korn, D. R. Stanski, eds., Drug Development Science: Obstacles and Opportunities for Collaboration Among Academia, Industry and Government, AAMC 2005, Available at: http:// services.aamc.org/Publications/showfile.cfm?file $=$ version 43 . pdf\&prd_id $=135 \&$ prv_id $=157$. Accessed 1/15/2008. 